\title{
Effects of gestational age and prenatal and perinatal events on the coagulation status in premature infants
}

\author{
M Salonvaara, P Riikonen, R Kekomäki, E Vahtera, E Mahlamäki, P Halonen, \\ K Heinonen
}

Arch Dis Child Fetal Neonatal Ed 2003;88:F319-F323

See end of article for authors' affiliations

Correspondence to:

Dr Salonvaara, Department

of Pediatrics, Kuopio

University Hospital, $\mathrm{POB}$

1777, FIN-70211 Kuopio,

Finland;

marjut.salonvaara@kuh.fi

Accepted

3 September 2002

\begin{abstract}
Objective: To study prospectively the effects of prematurity and perinatal events on the coagulation status of premature infants.

Patients and main outcome measures: Blood samples from premature infants born before 37 gestational weeks were taken for analysis of coagulation factors II, V, VII, and X and platelet count. Results: A total of 125 premature infants, 71 boys, were studied at the median postnatal age of 40 minutes (range 12-100). The lowest median activities of coagulation factors II, V, VII, and X and the platelet count were observed, as expected, in infants $(n=21)$ born at 24-27 weeks gestation. Twin B ( $n=14$ ) had lower median activities of coagulation factors II, V, VII, and X than twin A. Infants with evidence of mild asphyxia (Apgar score at 5 minutes $<7$ or cord $\mathrm{pH}<7.26$ ) had significantly $(p<0.05)$ lower levels of coagulation factors II, V, VII, and X and platelet counts than infants without asphyxia. Infants who were small for gestational age (SGA) had significantly $(p<0.05$ ) lower levels of coagulation factors $\mathrm{V}$ and $\mathrm{VII}$ and platelet counts than infants of appropriate size for gestational age. Other prenatal and perinatal variables examined (sex, maternal hypertension and/or pre-eclampsia, antenatal steroid use, mode of delivery, Apgar scores) did not show any significant associations with coagulation status, which may be explained by the small number of infants studied.

Conclusions: The data strongly suggest that there are distinct differences in specific coagulation tests in different patient populations, which could assist in the identification of extremely preterm, SGA, or asphyxiated preterm infants who may be susceptible to haemorrhagic problems perinatally.
\end{abstract}

T he study of coagulation status has particular importance for premature babies who are at risk of serious health problems. Haemorrhagic and/or thrombotic complications may increase morbidity and mortality in this age group. ${ }^{1}$

Andrew et al $^{12}$ found that the overall pattern of coagulation was similar in premature and full term infants. Concentrations of the vitamin $\mathrm{K}$ dependent factors have been shown to be significantly lower in fetuses and extremely premature infants than in term infants. ${ }^{3-5}$

However, the significance of and variation in the levels of the different components of the coagulation system for preterm babies born at 24-27 weeks gestation and 28-31 weeks gestation are not fully defined. ${ }^{6-8}$ Most of the current reference data for preterm infants born before 28 weeks gestation are obtained from fetuses. Coagulation data for extremely premature infants are not easy to obtain because of sampling difficulties after birth and the urgent need for intensive care.

Hypoxia and acidosis in particular have been shown to alter the coagulation status. ${ }^{90}$ Prenatal and perinatal risk factors, such as maternalmedication and morbidity, may disturb haemostasis and increase haemorrhagic incidents in the newborn. ${ }^{11}{ }^{12}$ In pre-eclampsia, activation of coagulation, and fibrinolytic and platelet systems may alter the haemostatic balance at birth and predispose to intravascular coagulation. ${ }^{13}$

The aims of our study were to determine the levels of the coagulation factors in premature infants at birth, and to assess the effects of gestational age and prenatal and perinatal events on the coagulation status.

\section{METHODS}

The study was carried out at the Department of Pediatrics, Kuopio University Hospital, from February 1996 to May 1998. The study protocol was approved by the research ethics com- mittee of Kuopio University Hospital, and informed written consent was obtained from parents before entering the study.

\section{Study design and patients}

All infants admitted to the neonatal intensive care unit in Kuopio University Hospital and born at less than 37 weeks gestation during the study period were eligible for the study. Gestational age was determined from maternal ultrasound examination before the end of 20 weeks gestation or the mother's last menstrual period. All infants received $1.0 \mathrm{mg}$ vitamin $\mathrm{K}$ intramuscularly immediately after birth. ${ }^{14}$

Patients were ineligible for the study if any of the following criteria were fulfilled: written informed consent was not obtained; blood samples for the study were not taken within two hours of birth; the infant had received intravenous infusions of heparin, blood, or plasma products or intravenous drugs before or concurrently with the taking of blood samples for the study. Thirty five children were excluded from the study because of protocol violation: written informed consent was not obtained in three cases, and blood samples were not taken within two hours of birth or the sampling failed in 32 cases. One baby, born at 27 weeks gestation, was dropped from the analysis of coagulation studies because of a distinct inconsistency between the coagulation factors, platelet count, and screening tests. This was thought to be due to an undefined mistake in the preanalytical phase of the study.

Table 1 gives the patient characteristics.

\section{Data collection}

The following data were collected prospectively according to the protocol: gestational age, birth weight, sex, mode of delivery, Apgar scores, and venous cord blood pH. Asphyxia was defined as venous cord blood $\mathrm{pH}<7.26$ or Apgar score $<7$ at 5 minutes. $\mathrm{pH}<7.0$ would provide a more robust diagnosis of pathological fetal acidaemia, but, because coagulation factors 
Table 1 Patient characteristics

\begin{tabular}{|c|c|c|c|c|c|c|c|c|c|}
\hline $\begin{array}{l}\text { Gestational } \\
\text { age (weeks) }\end{array}$ & $\mathrm{n}$ & Birth weight $(\mathrm{g})^{*}$ & $\begin{array}{l}\text { Male } \\
\text { n (\%) }\end{array}$ & $\begin{array}{l}\text { Caesarean } \\
\text { section } \mathrm{n}(\%)\end{array}$ & $\begin{array}{l}\text { Apgar score* at } \\
1 \mathrm{~min} / 5 \mathrm{~min}\end{array}$ & Cord vein $\mathrm{pH}^{*}$ & $\begin{array}{l}\text { SGA† } \\
\text { n (\%) }\end{array}$ & $\begin{array}{l}\text { Infants with } \\
\text { asphyxiał } \\
\text { n (\%) }\end{array}$ & $\begin{array}{l}\text { Ventilation } \\
\text { support } \\
\text { n (\%) }\end{array}$ \\
\hline $24-27$ & 21 & $790(515-1180)$ & 15 (71) & $16(76)$ & $6(2-9) / 8(3-9)$ & $7.31(7.02-7.43)$ & 9 (43) & $8(38)$ & $21(100)$ \\
\hline $28-30$ & 25 & 1150 (770-1850) & 10 (40) & $19(76)$ & 7 (2-9)/8(2-9) & 7.30 (6.90-7.50) & $7(28)$ & $9(36)$ & 23 (92) \\
\hline $31-33$ & 34 & 1820 (1 140-2710) & 21 (62) & 28 (82) & $8(3-9) / 9(6-10)$ & $7.31(7.08-7.48)$ & $10(30)$ & 7 (20) & $17(50)$ \\
\hline $34-36$ & 45 & 2460 (1670-3740) & $25(56)$ & $29(64)$ & $9(0-9) / 8(4-10)$ & 7.32 (6.89-7.48) & $13(29)$ & $10(22)$ & $17(38)$ \\
\hline
\end{tabular}

*Expressed as medians (range); †SGA, small for gestational age $\left(<-2.0\right.$ SD gestational age adjusted mean birth weight $\left.{ }^{15}\right)$; $\neq a s p h y x i a$ defined as venous cord blood $\mathrm{pH}<7.26$ or Apgar score $<7$ at 5 minutes.

are sensitive to hypoxia, we wished to include infants who may have been modestly hypoxaemic. Small for gestational age (SGA) was defined as a standardised birth weight $<-2.0$ standard deviations of the gestational age adjusted mean birth weight of ethnic growth curves. ${ }^{15}$ Ventilation support after birth was recorded.

The charts of the mothers were reviewed for information, including maternal medication and the presence or absence of pre-eclampsia or hypertension. Women who repeatedly had a systolic pressure $\geqslant 140 \mathrm{~mm} \mathrm{Hg}$ and a diastolic pressure $\geqslant 90$ $\mathrm{mm} \mathrm{Hg}$ were defined as having hypertension. Hypertension accompanied by proteinuria of at least $0.3 \mathrm{~g}$ per 24 hours was classified as pre-eclampsia.

Antenatal corticosteroid (betamethasone $12 \mathrm{mg}$ intramuscularly) was given to the mother if there was a risk of a premature birth before 35 gestational weeks. If necessary, buphenine hydrochloride infusion was given as a tocolytic agent to delay or prevent premature parturition. Antibiotics were used to treat diagnosed/suspected amnionitis or chorioamnionitis. The most common antihypertensive drugs for mothers were labetalol and nifedipine.

\section{Laboratory methods}

Tests for the coagulant activity of factors II, V, VII, and X, as well as prothrombin time, activated partial thromboplastin time, and platelet count, were used to evaluate the coagulation status of the preterm baby. As extremely premature babies were included in the study, the maximum volume of blood available for analysis was $3.5 \mathrm{ml}$, which was enough to determine factors II, V, VII, and X. Factors II, VII, and X are vitamin $\mathrm{K}$ dependent.

Factor II has the longest natural half life, and factor VII the shortest half life; thus they effectively reflect synthesis of vitamin $\mathrm{K}$ dependent coagulation factors in the liver. Factor $\mathrm{V}$ is synthesised very early in pregnancy compared with the other factors. Its synthesis is not dependent on vitamin $\mathrm{K}$, but it is labile and is consumed in disseminated intravascular coagulation, as well as is fibrinogen. Analysis of factor IX and fibrinogen would have needed another blood sample.

Samples for the assessment of coagulation status were obtained from the peripheral arterial or venous catheters immediately after placement. Blood samples for assay of coagulation factors flowed directly into Vacuette Greiner tubes $(1.8 \mathrm{ml})$. Samples for determining prothrombin time and activated partial thromboplastin time were drawn into special citrate tubes for infants (CTAD $0.1 \mathrm{ml}$; Becton Dickinson; $0.9 \mathrm{ml}$ ). The amount of anticoagulant was not adjusted for packed cell volume. However, median and range of packed cell volumes in each gestational age group were similar (data not shown). Samples for platelet count were taken into EDTA Microtainer (Becton Dickinson, Franklin Lakes, New Jersey, USA) tubes $(0.25 \mathrm{ml})$.

The blood for the coagulation studies was immediately centrifuged at room temperature at $1400 \mathrm{~g}$ for 30 minutes; plasma was removed, divided into aliquots, and kept at $-80^{\circ} \mathrm{C}$ for future assays. The coagulation factor samples were analysed batchwise in the Department of Hemostasis of the Finnish
Red Cross Blood Transfusion Service, Helsinki, Finland. Platelet count, prothrombin time, and activated partial thromboplastin time were determined immediately in the Department of Clinical Chemistry of Kuopio University Hospital, Finland.

The coagulant activity of factors II, V, VII, and X was determined by the one stage method with an STA Compact Analyser from Diagnostica Stago (Asnières, France). Factor deficient plasma and Neoplastine CI Plus were purchased from Diagnostica Stago. A frozen normal plasma pool was used as a standard, and calibrated with the international standard (National Institute for Biological Standards and Control, Potters Bar, Hertfordshire, UK) for prothrombin and factors VII and X. Activity was expressed as a percentage $(100 \%=1 \mathrm{IU} / \mathrm{ml})$.

Prothrombin time and activated partial thromboplastin time were measured with a Thrombolyzer (Behnk Elektronik, Norderstedt, Germany) using Thrombotest and Cephotest reagents (Nycomed, Oslo, Norway). Prothrombin time was expressed as the international normalised ratio (INR). Platelet counts were measured with a Coulter STKS analyzer (Coulter Corporation, Miami, Florida, USA).

\section{Statistical analysis}

Results are expressed as mean (SD) or median (range), as appropriate. Overall differences in the components between the gestational age groups were first tested with the KruskalWallis test. For each component measured, the pairwise comparisons between the gestational age groups were performed using the Mann-Whitney U test. Bonferroni correction was made for multiple comparisons. The Wilcoxon signed ranks test was used to analyse the coagulation factors and platelet count between twins A and B. Linear regression analysis was used to identify the associations between coagulation factors and the following prenatal and perinatal variables: sex, intrauterine growth status, maternal hypertension and/or preeclampsia, antenatal steroid use, mode of delivery, gestational age, Apgar scores, and venous cord blood pH. p $<0.05$ were considered significant. SPSS (9.0 for Windows) was used for all data analysis.

\section{RESULTS}

The series comprised 125 children, 71 boys, recruited during 28 months. The median gestational age was 33 weeks (range 24-36), and the median birth weight was 1740 g (range 5153740 ). There were 88 singletons, 14 sets of twins, and three sets of triplets. Thirty nine (31\%) were SGA. There were 34 $(27 \%)$ asphyxiated infants; 15 of them were also growth retarded. The newborns were divided into four groups on the basis of gestational age: group 1, 21 (17\%) babies with gestational age $<28$ weeks; group 2, $25(20 \%)$ babies with gestational age 28-30 weeks; group 3, 34 (27\%) babies with gestational age 31-33 weeks; group 4, 45 (36\%) babies with gestational age 34-36 weeks. These infants represent $98 \%$ of all infants born before 28 weeks, $71 \%$ of infants born at $28-30$ weeks, $62 \%$ of infants born at $31-33$ weeks, and $18 \%$ of infants born at 34-36 weeks during the study period. 
Table 2 Coagulation status in each gestational age group within two hours of birth

\begin{tabular}{|c|c|c|c|c|c|c|c|c|}
\hline Gestational age (weeks) & $n$ & Factor II (\%) & Factor V (\%) & Factor VII (\%) & Factor X (\%) & $\begin{array}{l}\text { Platelets ( } \\
\left.10^{\circ} / /\right)\end{array}$ & APTT† (s) & PT-INR + \\
\hline $24-27$ & 21 & & & & & & & \\
\hline Mean (SD) & & $31(9)^{*}$ & $57(20)^{*}$ & $43(16) *$ & $35(10)$ & $181(71)^{*}$ & $49(8)$ & $1.4(0.3)$ \\
\hline Median & & 30 & 61 & 46 & 34 & 166 & 48 & 1.4 \\
\hline Range & & $20-49$ & $10-102$ & $14-81$ & $23-56$ & $77-338$ & $28-36$ & $1.1-2.0$ \\
\hline $28-30$ & 25 & & & & & & & \\
\hline Mean (SD) & & $30(8)^{*}$ & $67(25)$ & $50(22)^{*}$ & $36(9)$ & $214(64)^{*}$ & $46(10)$ & $1.4(0.2)$ \\
\hline Median & & 29 & 65 & 51 & 34 & 225 & 45 & 1.3 \\
\hline Range & & $17-47$ & $29-113$ & $15-103$ & $20-55$ & $59-322$ & $30-72$ & $1.0-2.2$ \\
\hline $31-33$ & 34 & & & & & & & \\
\hline Mean (SD) & & $39(13)$ & $78(28)$ & $64(28)$ & $44(15)$ & $237(34)$ & $44(22)$ & $1.4(0.7)$ \\
\hline Median & & 37 & 73 & 62 & 42 & 238 & 41 & \\
\hline Range & & $13-64$ & $5-136$ & $11-134$ & $9-72$ & $59-416$ & $28-145$ & $0.9-4.5$ \\
\hline $34-36$ & 45 & & & & & & & \\
\hline Mean (SD) & & $38(10)$ & $76(26)$ & $63(23)$ & $40(10)$ & $255(45)$ & $46(28)$ & $1.3(0.3)$ \\
\hline Median & & 37 & 73 & 63 & 39 & 261 & 41 & 1.3 \\
\hline Range & & $13-64$ & $17-143$ & $12-114$ & $22-63$ & $52-434$ & $27-200$ & $1.1-2.7$ \\
\hline Term infant $\ddagger$ & & $48(11)$ & $72(18)$ & 66 (19) & 40 (14) & & $43(6)$ & \\
\hline
\end{tabular}

${ }^{*} p<0.05$ compared with gestational age 34-36 weeks; †Data available for 101 infants; $\ddagger$ at the age of 1 day.

APTT, Activated partial thromboplastin time; PT, prothrombin time; INR, international normalised ratio.

Table 3 Coagulation status in asphyxiated versus non-asphyxiated infants and SGA versus AGA infants within two hours of birth

\begin{tabular}{|c|c|c|c|c|c|c|c|c|}
\hline Infant group & $\mathrm{n}$ & Factor II (\%) & Factor V (\%) & Factor VII (\%) & Factor X (\%) & $\begin{array}{l}\text { Platelets }(\times \\
\left.10^{\circ} / \mathrm{I}\right)\end{array}$ & APTT‡ (s) & PT-INR \\
\hline Asphyxiated & 34 & $30(10)^{*}$ & $61(27)$ * & $44(27)$ * & $32(10)^{*}$ & $202(87)^{*}$ & $50(25)$ & $1.6(0.6)^{*}$ \\
\hline Non-asphyxiated & 91 & 38 (11) & $75(25)$ & $62(24)$ & 42 (11) & 239 (70) & $45(20)$ & $1.3(0.3)$ \\
\hline SGA & 39 & 35 (11) & $63(25) \dagger$ & $47(23) \dagger$ & 38 (12) & $182(60) \dagger$ & $49(30)$ & $1.5(0.4)$ \\
\hline AGA & 86 & 36 (11) & $76(26)$ & $62(23)$ & 40 (11) & $250(74)$ & 45 (17) & $1.3(0.4)$ \\
\hline
\end{tabular}

Values are mean (SD).

${ }^{*} p<0.05$, compared with non-asphyxiated infants; $\dagger p<0.05$, compared with AGA; $\ddagger$ Data available for 101 infants.

APTT, Activated partial thromboplastin time; PT, prothrombin time; INR, international normalised ratio; SGA, small for gestational age; AGA, appropriate size for gestational age.

The median time interval between birth and blood sampling was 40 minutes (range 12-100). The lowest median levels of coagulation factors II, V, VII, and $X$ and platelet count were detected in infants $(\mathrm{n}=21)$ born at $24-27$ weeks gestation. The levels increased towards term infant reference data with increasing gestational age. The levels of factors II, V, and VII were significantly different between infants born at 24-27 weeks gestation $(\mathrm{n}=21)$ and those born at 34-36 weeks gestation $(\mathrm{n}=45)$ (table 2$)$. The infants born at 24-27 weeks gestation had a significantly lower median platelet count than those born at 34-36 weeks gestation. No difference was found in activated partial thromboplastin time and INR values between the gestational groups (table 2).

Infants with birth asphyxia had lower mean concentrations of coagulation factors, platelet counts, and INR than infants without asphyxia (table 3). SGA infants had lower mean concentrations of coagulation factors V and VII and platelet count (table 3).

In twin pregnancies $(\mathrm{n}=14)$, twin $\mathrm{B}$ had lower median activities of coagulation factors II, V, VII, and X than twin A (fig 1). A-B difference between the twin pairs showed that twin A had consistently higher coagulation factor activities than twin B (table 4). Median platelet count did not differ between the twins $\left(235 \times 10^{9} / 1 \vee 240 \times 10^{9} / 1\right)$. There were no differences in birth weight, Apgar score, or cord blood $\mathrm{pH}$ between twins.

Linear regression analysis showed that asphyxiated infants had significantly lower activities of coagulation factors II, V, VII, and $X$ and platelet counts than non-asphyxiated infants. SGA infants had significantly $(\mathrm{p}<0.05)$ lower activities of coagulation factors V and VII and platelet counts than appropriate for gestational age infants. Other prenatal and perinatal

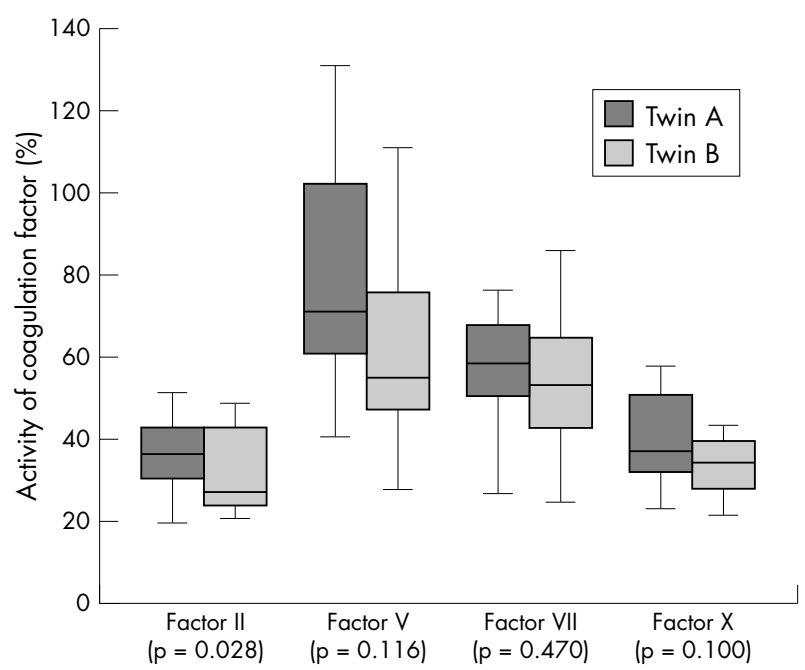

Figure 1 Median coagulation factor activities with the 25 th and 75th quartile ranges (boxes) with extreme values of each coagulation factor in twin pairs $(n=14)$. Wilcoxon signed ranks test was used to analyse the coagulation factors.

variables examined (sex, maternal hypertension and/or pre-eclampsia, antenatal steroid used, mode of delivery, Apgar scores) did not show any significant associations with coagulation status. Gestational age, intrauterine growth status, and venous cord blood pH did not affect the haemostasis screening tests in linear regression analysis. 
Table 4 Differences in the coagulation factor activities between the twin pairs $(n=14)$

\begin{tabular}{ll}
\hline Coagulation factor & $\begin{array}{l}\text { Difference between twin A and } \\
\text { twin B }\end{array}$ \\
\hline Factor II & $4.5(-7$ to 17$)$ \\
Factor V & $9(-27$ to 84$)$ \\
Factor VII & $1.5(-23$ to 49$)$ \\
Factor X & $5(-11$ to 23$)$ \\
\hline
\end{tabular}

Data given as median (range).

Antenatal medication of the mothers $(\mathrm{n}=106)$ was as follows: 19 (18\%) mothers received no drugs; 22 (21\%) mothers received antibiotics before delivery; 54 (51\%) mothers and 71 newborns (57\%) were exposed to corticosteroids; 25 (24\%) mothers had antihypertensive drugs. Antenatal exposure to antihypertensive drugs and/or corticosteroids did not affect coagulation factor activity or the haemostasis screening tests.

\section{DISCUSSION}

The purpose of this study was to investigate the coagulation factors of premature infants at birth and to assess the effects of prenatal and perinatal events on the coagulation status. Predictably, our evaluation showed that extremely premature infants have lowest median activities of coagulation factors, and SGA and asphyxiated preterms have decreased mean activities of coagulation factors favouring bleeding tendencies. We found that coagulation screening tests are insensitive in picking up coagulation defects in preterm infants. Abnormal screening tests strongly support coagulopathy, such as disseminated intravascular coagulation, but normal results do not rule out activation of the coagulation and fibrinolytic systems. Screening tests could be useful in preterm infants if there is a clinical suspicion of coagulopathy. Maternal medication or antenatal exposure to corticosteroids did not affect the measured coagulation status.

The haemostatic system evolves gradually throughout gestation and early infancy. ${ }^{12}$ The newborn physiological status affects the risk for and presence of acquired haemostatic disorders. This may make congenital haemostatic disorders more difficult to diagnose. In particular, prematurity, birth asphyxia, hypoxia, and intrauterine growth retardation have been associated with haemostatic abnormalities. ${ }^{716}$ The present study confirms previous observations that coagulation factor activity and platelet counts in extremely preterm infants are lower than in those born at 34-36 weeks. ${ }^{1-6}$

The haemostatic system of extremely preterm infants is not well understood. One reason may be the difficulty of obtaining sufficient amounts of blood for tests. In this study, blood samples were withdrawn through peripheral arterial or venous catheters before any drugs were given to the newborn. The correct sampling technique and appropriate handling of the specimen increases the reliability of the results. In an optimal setting, broad testing of haemostatic factors-for example, coagulation and fibrinolytic as well as prothrombotic factorswould be desirable.

As far as we know, no comparative information about the specific coagulation factor activities in twins is available. We evaluated the coagulation studies of 14 pairs of twins, who did not differ from each other in birth weight, Apgar scores, or cord blood $\mathrm{pH}$. Twin B had lower levels of the coagulation factors II, V, VII, and X than twin A, but no differences were found in the platelet counts. There was no difference in the time interval from birth to blood sampling between twin $\mathrm{A}$ and twin B. A hypercoagulable state with consumption in placental circulation may be one reason why the levels of coagulation factors were low in twin $\mathrm{B}$.
Pre-eclampsia is known to be associated with a hypercoagulable state in both maternal and placental circulations. ${ }^{17-19}$ The primary defect is speculated to occur at mid-trimester. Severe pre-eclampsia is one of the most common reasons for intrauterine growth retardation. A hypercoagulable state with consumption may be among the reasons why the coagulation factors and the platelet counts were low in our SGA children.

Thrombopoietin is a potent megakaryocyte colony stimulating factor. In thrombocytopenia associated with intrauterine growth retardation or pregnancy induced hypertension, infants have decreased megakaryocyte mass. ${ }^{20}$ Preterm babies may have impaired ability to increase thrombopoietin concentrations in response to thrombocytopenia. ${ }^{20}$ However, the exact mechanisms of this regulation are not known, although megakaryocytes are known to be susceptible to asphyxia. ${ }^{21}{ }^{22}$ The present study confirms that platelet counts in infants with birth asphyxia are low, which may further increase the vulnerability of the haemostatic system.

Not only does the generation of thrombin, a critical enzyme in haemostasis, differ profoundly during fetal and early neonatal life, but also thrombin activity is lower in premature infants than in adults. ${ }^{23}$ The altered regulation of thrombin generation is at least partly due to the presence of low levels of the vitamin $\mathrm{K}$ dependent procoagulants (factors II, VII, IX, and X) and the contact factors XI and XII. ${ }^{23}$ Despite the lower capacity to generate thrombin, there is no clinical evidence that healthy infants have a higher risk of bleeding complications. However, the further reduction in coagulation factor levels seen in sick and premature infants may contribute to the haemorrhagic complications. ${ }^{7}$

Haemostasis of a premature baby is a dynamic system that gradually evolves towards term. However, when prematurity is associated with asphyxia and/or intrauterine growth retardation, alterations in the concentrations of the coagulation factors may cause an imbalance and thus predispose the infant at risk to haemorrhagic or thromboembolic complications.

As the fibrinolytic system was not evaluated in this study, it is difficult to estimate to what extent haemostatic defects are balanced by reduced fibrinolysis or increased thrombotic risk. Preterm babies with intrauterine growth retardation and/or asphyxia may need more careful investigation for haemorrhagic symptoms after birth. These high risk neonates should be properly evaluated during neonatal intensive care. Good perinatal care of preterm twins and triplets should be emphasised. Further research is needed to determine whether these findings have relevance to haemorrhagic problems of extremely preterm infants.

\section{ACKNOWLEDGEMENTS}

This study was financially supported by the Finnish Pediatric Research Foundation and the State Research Fund for University Hospitals.

Authors' affiliations

M Salonvaara, P Riikonen, K Heinonen, Department of Pediatrics, Kuopio University Hospital and Kuopio University, Kuopio, Finland R Kekomäki, E Vahtera, Finnish Red Cross Blood Transfusion Service, Helsinki, Finland

E Mahlamäki, Department of Clinical Chemistry, Kuopio University Hospital

P Halonen, Computing Center, University of Kuopio

\section{REFERENCES}

1 Andrew M, Paes B, Milner R, et al. Development of the human coagulation system in the full-term infant. Blood 1987;70:165-72.

2 Andrew M, Paes B, Milner R, et al. Development of the human coagulation system in the healthy premature infant. Blood 1988;7:1651-7

3 Heikinheimo R. Coagulation studies with fetal blood. Biol Neonate 1964;7:319-24.

4 Bleyer WA, Hakami N, Shepard TH. The development of hemostasis in the human fetus and newborn infant. J Pediatr 1971;79:838-53. 
5 Barnard DR, Simmons MA, Hathaway WE. Coagulation studies in extremely premature infants. Pediatr Res 1979;13:1330-5.

6 Forestier F, Daffos F, Galacteros F, et al. Hematological values of 163 normal fetuses between 18 and 30 weeks of gestation. Pediatr Res 1986;20:342-6.

7 Andrew M, Paes B, Johnston M. Development of the hemostatic system in the neonate and young infant. Am J Pediatr Hematol Oncol 1990; 12:95-104.

8 Montgomery RR, Marlar RA, Gill JC. Newborn haemostasis. Clin Haematol 1985; 14:443-60.

9 Andrew $M, \mathrm{O}^{\prime}$ Brodovich $\mathrm{H}$, Mitchell L. Fetal lamb coagulation system during birth asphyxia. Am J Hematol 1988;28:201-3.

10 Mautone A, Giordano P, Montagna O, et al. Coagulation and fibrinolytic systems in the ill preterm newborn. Acta Paediatr 1997:86:1100-4.

11 Shankaran S, Baver CR, Bain R, et al. Prenatal and perinatal risk and protective factors for neonatal intracranial hemorrhage. Arch Pediatr Adolesc Med 1996;150:491-7.

12 Shapiro AD, Jacobson L, Armon ME, et al. Vitamin K deficiency in the newborn infant: prevalence and perinatal risk factors. J Pediatr 1986;109:675-80

13 Dati F, Pelzer H, Wagner C. Relevance of markers of hemostasis activation in obstetrics/gynecology and pediatrics. Semin Thromb Hemost 1998;24:443-8.
14 Report of Committee on Nutrition, American Academy of Pediatrics. Vitamin K compounds and the water-soluble analogues: use in therapy and prophylaxis in pediatrics. Pediatrics 1961;28:501-7.

15 Pihkala J, Hakala T, Voutilainen P, et al. Characteristics of recent fetal growth curves in Finland. Duodecim 1989;105:1540-6.

16 Chessells JM, Wigglesworth JS. Coagulation studies in severe birth asphyxia. Arch Dis Child 1971;46:253-6.

17 Beardsley DS. Hemostasis in the perinatal period: approach to the diagnosis of coagulation disorders. Semin Perinat 1991;15:25-34.

18 Condie RG. Components of the haemostatic mechanism at birth in pre-eclampsia with particular reference to fetal growth retardation. $\mathrm{Br} J$ Obstet Gynaecol 1976;83:943-7.

19 Lox CD, Word RA, Corrican JJ. Effects of preeclampsia on maternal and cord blood clotting activity. Am J Perinatol 1985;2:279-82.

20 Watts TL, Murray NA, Roberts IA. Thrombopoietin has a primary role in the regulation of platelet production in preterm babies. Pediatr Res 1999;46:28-32.

21 Andrew M, Kelton J. Neonatal thrombocytopenia. Clin Perinatol 1984;11:359-91.

22 Buchanan GR. Coagulation disorders in the neonate. Pediatr Clin North Am 1986:33:203-20

23 Andrew M, Schmidt B, Mitchell L, et al. Thrombin generation in newborn plasma is critically dependent on the concentration of prothrombin. Thromb Haemost 1990;63:27-30.

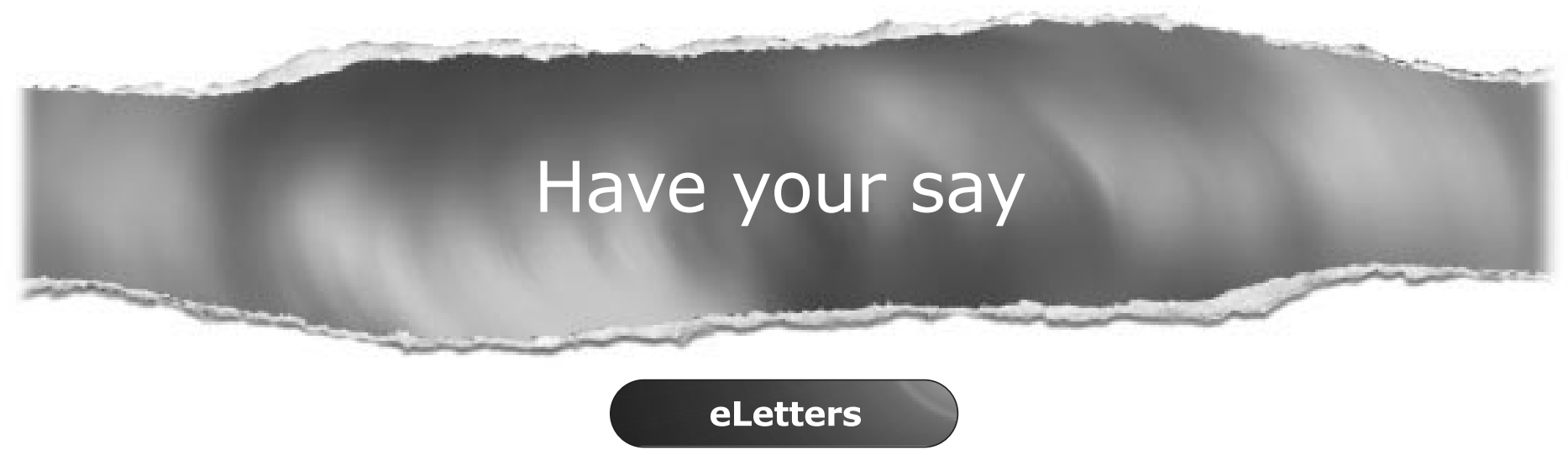

If you wish to comment on any article published in Archives of Disease in Childhood you can send an eLetter using the eLetters link at the beginning of each article. Your response will be posted on Archives of Disease in Childhood online within a few days of receipt (subject to editorial screening).

www.archdischild.com 